\section{CBT does not improve relapse rates in people with recently relapsed psychosis}

\section{QUESTION}

Question: What is the effect of cognitive behavioural therapy (CBT) and family intervention on relapse rates and symptoms in people with recently relapsed non-affective psychosis?

Patients: 301 people aged $18-65$ years (218 without carers, 83 with carers) with non-affective psychosis (ICD-10 category F2 and DSM-IV) and a second or subsequent psychotic episode not more than 3 months before the trial began, plus a rating of at least 4 for one or more positive symptoms on the Positive and Negative Syndrome Scale (PANSS). Exclusion criteria: primary diagnosis of alcohol or substance dependency; intellectual disability or organic syndrome; unreliable residential arrangements; or limited spoken English.

Setting: Five local mental health services, UK; recruitment 2002-2004.

Intervention: People with carers randomly allocated to: CBT for psychosis (12-20 sessions over 9 months, targeting key aspects of relapse prevention) plus treatment as usual (TAU), family intervention plus TAU or TAU only. People without carers randomly allocated to: the same CBT regimen plus TAU or TAU only. Quality of and adherence to the CBT protocol was assessed.

Outcomes: Primary outcomes: relapse (re-emergence of, or significant deterioration in, positive psychotic symptoms of at least moderate severity, persisting for $\geqslant 2$ weeks) and total days in hospital. Secondary outcomes: psychotic symptom measures (PANSS); delusions (Psychotic Symptom Rating Scale (PSYRATS)); hallucinations (PSYRATS); depression (Beck Depression Inventory Second Edition (BDI-II)); and social functioning (SOFAS score). Assessments took place at 3, 6, 12 and 24 months.

Patient follow-up: At 24 months, $96 \%$ of participants were analysed for primary and $80 \%$ for secondary outcomes.

\section{METHODS}

Design: Randomised controlled trial (stratified by carer, centre and inpatient or outpatient status at time of relapse). Allocation: Concealed.

Blinding: All treatments were single blind (assessors blind). Follow-up period: 24 months.

\section{MAIN RESULTS}

There were no differences in remission, relapse or total days spent in hospitals among the treatment groups at 12 or 24 months (see online table). At 24 months, CBT improved depression compared with TAU (mean difference in BDI-II score $-3.07,95 \%$ CI -6.04 to $-0.11 ; p<0.05$; results pooled for those with and without carers).

\section{CONCLUSIONS}

Neither CBT nor family therapy improve relapse rates or reduces the number of days in hospital for people with psychosis who have recently relapsed.

\section{NOTES}

As only a low proportion (less than 50\%) of participants showed full remission (absence of positive symptoms) from their initial episode, months in full or partial remission (improvement in or absence of positive symptoms) was used as an additional indicator of the primary outcome. Authors suggest treating significant results in secondary outcomes with caution because of the risk of type I errors from multiple significance testing.

\section{ABSTRACTED FROM}

Garety PA, Fowler DG, Freeman D, et al. Cognitivebehavioural therapy and family intervention for relapse prevention and symptom reduction in psychosis: randomized controlled trial. Br J Psychiatry 2008;192:412-23.

Correspondence to: Professor Philippa Garety, Department of Psychology, P077, Institute of Psychiatry, De Crespigny Pk, London SE5 8AF, UK; p.garety@iop.kcl.ac.uk

Source of funding: Wellcome Trust Programme Grant.

- A table is published online only at http://ebmh.bmj.com/content/vol12/issue1
$R$ elapse rates in psychosis remain high despite advances in neuroleptics and psychological intervention. They remain particularly high (37-55\%) in people living alone compared with those with carers $(21-28 \%)$, a finding of significant interest in this paper. The clinical challenges presented by this population are well illustrated by the adverse events recorded over the course of the trial -22 serious suicide attempts and 45 violent incidents among the 301 participants. Both the difficulty and value of more effective intervention in this field are readily apparent. ${ }^{1}$ Disappointingly, cognitive behavioural therapy (CBT) based interventions look to be no better than good quality treatment as usual (TAU). The limitations of CBT are most clearly evident in individual CBT in which well trained and experienced therapists were judged to have delivered competent therapy that adhered to the treatment manual. Thus although individual CBT for persistent psychotic symptoms when added to medication may confer some modest benefits in reducing emotional distress, ${ }^{2}$ and should continue to be offered, it is not efficacious in preventing further relapse among those recovering from a recent relapse. Given the scarcity of therapist resources in this area, this is a helpful piece of evidence for service planning and, to their credit, it is a conclusion that the authors propose without qualification.

The inadequately powered comparison of family intervention with TAU, in which relapse rates across all three conditions were relatively low, should perhaps be interpreted more cautiously. The challenge here is to persuade the families most in need of help to participate and to stay the course. In this connection it is worth noting that $56 \%$ of 683 patients who satisfied inclusion criteria refused to consent to randomisation and of those who did participate, a significant minority (25-30\%) dropped out of therapy before any helpful learning could take place. But what are these learning processes and how can CBT therapists better engage people with psychosis in learning to prevent relapse? This paper has no answer to these important questions but it does provide us with an exemplary model of how to conduct a rigorous, multicentre clinical trial that gives a clear and unambiguous guide to the limitations of current treatment technology.

Robert C Durham, PhD, FBPsS

University of Dundee, Dundee, UK

Competing interests: None.

1. Thornicroft G, Tansella M, Becker T, et al. The personal impact of schizophrenia in Europe. Schizophr Res 2004:69:125-32.

2. Wykes T, Steel C, Everitt B, et al. Cognitive behaviour therapy for schizophrenia: effect sizes, clinical models and methodological rigor. Schizophr Bull 2008;34:523-37. 